\title{
Combining affinity propagation clustering and mutual information network to investigate key genes in fibroid
}

\author{
QIAN-SONG CHEN ${ }^{1}$, DAN WANG ${ }^{1}$, BAO-LIAN LIU ${ }^{2}$, SHU-FENG GAO ${ }^{1}$, DAN-LI GAO ${ }^{1}$ and GUI-RONG LI ${ }^{1}$ \\ Departments of ${ }^{1}$ Gynaecology and ${ }^{2}$ Reproductive Genetics, Tangshan Maternal and \\ Child Healthcare Hospital, Tangshan, Hebei 063000, P.R. China
}

Received December 9, 2015; Accepted February 1, 2017

DOI: $10.3892 /$ etm.2017.4481

\begin{abstract}
The aim of the present study was to investigate key genes in fibroids based on the multiple affinity propogation-Krzanowski and Lai (mAP-KL) method, which included the maxT multiple hypothesis, Krzanowski and Lai (KL) cluster quality index, affinity propagation (AP) clustering algorithm and mutual information network (MIN) constructed by the context likelihood of relatedness (CLR) algorithm. In order to achieve this goal, mAP-KL was initially implemented to investigate exemplars in fibroid, and the maxT function was employed to rank the genes of training and test sets, and the top 200 genes were obtained for further study. In addition, the KL cluster index was applied to determine the quantity of clusters and the AP clustering algorithm was conducted to identify the clusters and their exemplars. Subsequently, the support vector machine (SVM) model was selected to evaluate the classification performance of mAP-KL. Finally, topological properties (degree, closeness, betweenness and transitivity) of exemplars in MIN constructed according to the CLR algorithm were assessed to investigate key genes in fibroid. The SVM model validated that the classification between normal controls and fibroid patients by mAP-KL had a good performance. A total of 9 clusters and exemplars were identified based on $\mathrm{mAP}-\mathrm{KL}$, which were comprised of CALCOCO2, COL4A2, COPS8, SNCG, PA2G4, C17orf70, MARK3, BTNL3 and TBC1D13. By accessing the topological analysis for exemplars in MIN, SNCG and COL $4 A 2$ were identified as the two most significant genes of four types of methods, and they were denoted as key genes in the progress of fibroid. In conclusion, two key genes (SNCG and COL4A2) and 9 exemplars
\end{abstract}

Correspondence to: Dr Bao-Lian Liu, Department of Reproductive Genetics, Tangshan Maternal and Child Healthcare Hospital, 14 Jianshe South Road, Tangshan, Hebei 063000, P.R. China E-mail: baolianliu2015@yeah.net

Key words: fibroid, cluster, affinity propagation, mutual information network, gene were successfully investigated, and these may be potential biomarkers for the detection and treatment of fibroid.

\section{Introduction}

Affinity propagation (AP) is a relatively new clustering algorithm that was introduced by Frey and Dueck (1) to determine a so-called exemplar for clustered data, which is a sample that is most representative for the respective cluster. It works for any meaningful measure of similarity between data samples, but does not require a vector space structure and the exemplars are selected amongst the data samples observed and not computed as hypothetical averages of cluster samples (2). These advantages make AP clustering particularly suitable for applications in bioinformatics, which has been verified by previously published studies (3-5). For instance, Kiddle et al (3) successfully revealed transcriptional modules in Arabidopsis thaliana based on temporal clustering of gene expression data by AP.

Prior to controlling the quality of the partition of a known number of clusters with AP, Sakellariou et al (6) supplemented the Krzanowski and Lai (KL) index (7) to evaluate the optimum number of clusters, by retaining maxT function in order to rank the genes in microarray data. This combination offers a more meaningful way of investigating exemplars or informative genes for disease and the relative target treatment. However, genes typically work together with other genes in complex processes associated with tumors. A network-based approach is capable of extracting informative and notable genes dependent on biomolecular networks. For instance, a protein-protein interaction network, co-expression network and mutual information network (MIN) rather than individual genes $(8,9)$.

Therefore, the present study combined multiple (m) AP-KL and MIN to investigate key genes in fibroids, which made the results, more reliable. $\mathrm{mAP}-\mathrm{KL}$ was initially implemented to investigate clusters and exemplars in fibroid, and the support vector machines (SVMs) model was selected to evaluate the classification performance of mAP-KL. MIN for cluster genes was constructed based on the context likelihood of relatedness (CLR) algorithm, and topological analysis (degree, closeness, betweenness and transitivity) of exemplars was performed to investigate key genes in fibroid. Key genes may be potential biomarkers for further prognostic and therapeutic insights for fibroid. 


\section{Materials and methods}

Microarray data. In the present study, the gene expression data for the fibroid (access number E-GEOD-64763) originated from the A-AFFY-37-Affymetrix GeneChip Human Genome U133A 2.0 [HG-U133A_2] Platform of the ArrayExpress database (ebi.ac.uk/arrayexpress/), and shared a set of 25 fibroid samples that had been compared to 29 normal controls. The total samples were divided into two sets according to a ratio of $3: 2$, and 32 were kept to build a balanced training set (16 fibroid and 16 normal samples). In total, 22 were used to construct a test set for the purpose of validating the classification models (9 fibroid and 13 normal samples). In E-GEOD-64763, a total of 22,277 probes were detected.

To further control the quality of data and eliminate batch effects caused by experimental parameters and other factors, all data underwent mean-centering (10), z-score (11), quantile (12) and cyclic loess (13) normalization across samples, and $\log 2$ transformation was subsequently performed on the normalized data. The preprocessed results are illustrated in Fig. 1 and a better association was identified between the density and intensity of genes following cyclic loess preprocessing compared with that of raw data and other methods. Therefore, the preprocessed training set and test set data underwent further analysis for fibroid.

$m A P-K L$. A data-driven and classifier-independent hybrid feature selection method was implemented, mAP-KL, which included maxT multiple hypothesis testing (14), KL cluster quality index (7) and the AP clustering algorithm (1), in order to select a small subset of informative genes of fibroid. The hypothesis was that among the statistically significant genes there should be clusters of genes that share similar biological functions correlated with the disease investigated, thus, instead of keeping a number of the top ranked genes, it would be more appropriate to define and keep a number of gene cluster exemplars (6). Subsequently, the index of KL was applied to determine the number of clusters solely on the fibroid samples of the training test set. Finally, the AP clustering method was engaged to detect clusters and provide a list of the most informative genes of each cluster, the so-called exemplars.

MaxT hypothesis testing. In the present study, the maxT function, which computes permutation adjusted P-values for step-down multiple testing procedures (15), was employed to rank the genes of the training set and the top $N$ genes were then reserved for further exploitation (16). The family-wise error rate was evaluated in order to correct P-values using the Wilcoxon rank sum statistic with permuted class labels. Furthermore, the maximum Wilcoxon statistic was recorded for 1,000 random permutations and the P-value for each gene was estimated as the proportion of the maximum permutation-based $t$-statistics that were greater than the observed value (17).

$K L$ cluster quality index. Prior to clustering analysis with AP, KL as induced in the ClusterSim package version 0.45-1 (University of Economics, Wroclaw, Poland) was utilized to define the number of clusters, which in essence would be the number of representative genes. The KL was calculated by the following formula:
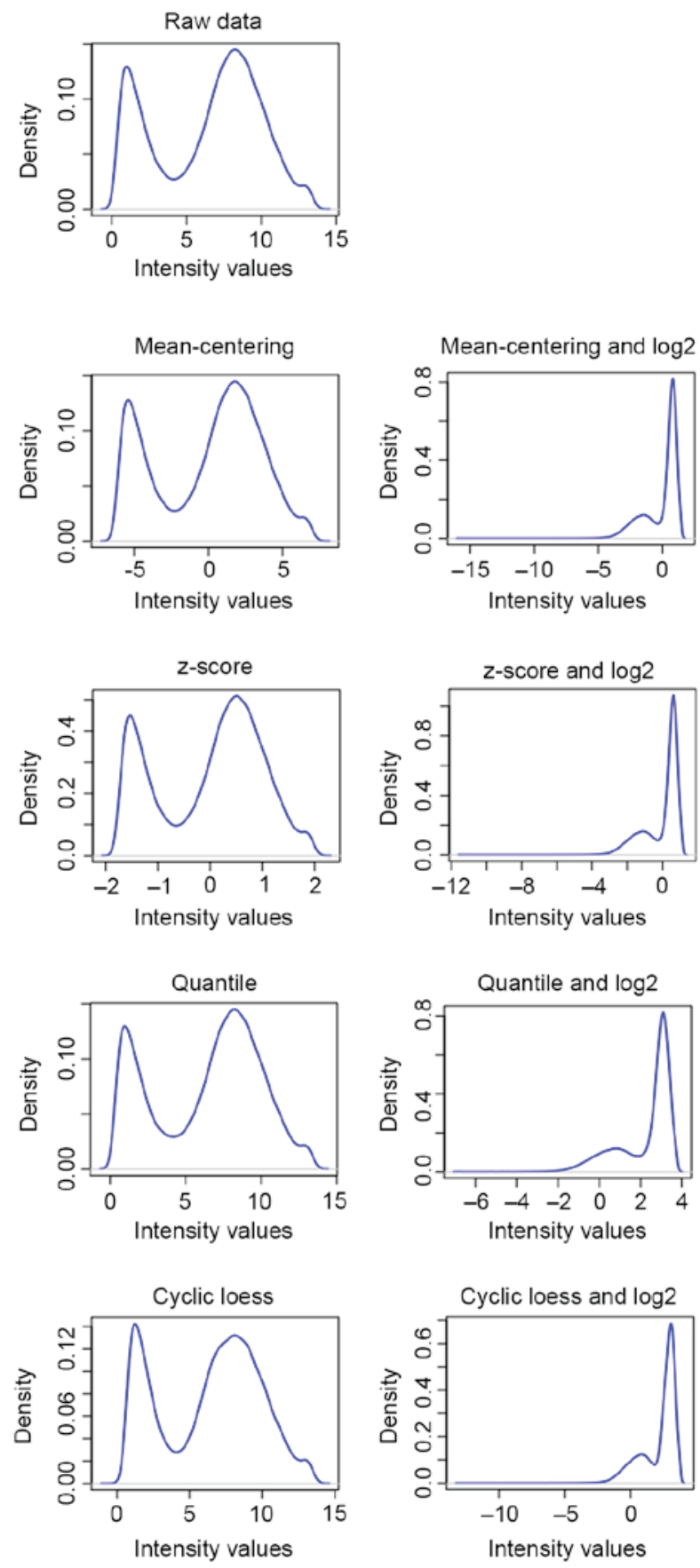

Figure 1. Preprocessing for microarray data by mean centering, z-score, quantile and cyclic loess methods.

$$
K L(k)=\mid \frac{\emptyset(k)}{\emptyset(k+1)}
$$

Of which $\emptyset(k)=(k-1)^{2 / P} W_{k-1}-k^{2 / P} W_{k}$

Where $k$ was the number of clusters and $W_{\mathrm{k}}$ represented the within-cluster sum of squared errors. The clusters that met a threshold of gene numbers $<50$ were regarded as KL clusters.

AP clustering algorithm. An AP clustering method included in the APCluster package version 1.3.2 (Johannes Kepler 
Table I. Top 100 genes based on maxT multiple hypothesis testing.

\begin{tabular}{|c|c|}
\hline Number & Gene \\
\hline 1 & IGSF3 \\
\hline 2 & $N A V 2$ \\
\hline 3 & KIF 5C \\
\hline 4 & CAPN6 \\
\hline 5 & FMO4 \\
\hline 6 & $B A X$ \\
\hline 7 & EPB41L2 \\
\hline 8 & KCNS3 \\
\hline 9 & COLAAI \\
\hline 10 & $S Y B U$ \\
\hline 11 & GPSM2 \\
\hline 12 & ABLIM3 \\
\hline 13 & CAMKMT \\
\hline 14 & POPDC2 \\
\hline 15 & $M A P 4 K 4$ \\
\hline 16 & CALCOCO2 \\
\hline 17 & TIMM44 \\
\hline 18 & $N P D C 1$ \\
\hline 19 & HEPH \\
\hline 20 & ANXA1 \\
\hline 21 & COLAA2 \\
\hline 22 & KCNN1 \\
\hline 23 & PIK3RI \\
\hline 24 & COPS8 \\
\hline 25 & $B C A N$ \\
\hline 26 & ITM2C \\
\hline 27 & C9orf3 \\
\hline 28 & DCHS1 \\
\hline 29 & $A D G R L 1$ \\
\hline 30 & $A L D H 2$ \\
\hline 31 & РНYНIР \\
\hline 32 & $F A M 63 B$ \\
\hline 33 & CYR61 \\
\hline 34 & $L G A L S 3$ \\
\hline 35 & $B C A N$ \\
\hline 36 & $S N C G$ \\
\hline 37 & PLAT \\
\hline 38 & $U S T$ \\
\hline 39 & $V A M P 2$ \\
\hline 40 & $S A V 1$ \\
\hline 41 & COL9A2 \\
\hline 42 & RPL38 \\
\hline 43 & GATA2 \\
\hline 44 & TEX10 \\
\hline 45 & $I R F 2$ \\
\hline 46 & $C B X 8$ \\
\hline 47 & MST1 \\
\hline 48 & PRR5L \\
\hline 49 & $P X D N$ \\
\hline 50 & PRKDI \\
\hline 51 & PEX7 \\
\hline 52 & TMEM246 \\
\hline
\end{tabular}

Table I. Continued.

\begin{tabular}{|c|c|}
\hline Number & Gene \\
\hline 53 & $E G F R$ \\
\hline 54 & HTATIP2 \\
\hline 55 & $P D G F C$ \\
\hline 56 & $P C B P 2$ \\
\hline 57 & SULT1A1 \\
\hline 58 & GCN1L1 \\
\hline 59 & COL5A2 \\
\hline 60 & COPS8 \\
\hline 61 & $F B X L 7$ \\
\hline 62 & $L M B R D 1$ \\
\hline 63 & $D P T$ \\
\hline 64 & PCNA \\
\hline 65 & ADGRE5 \\
\hline 66 & $P A R V B$ \\
\hline 67 & SULT1A2 \\
\hline 68 & $A C A N$ \\
\hline 69 & $K L F 4$ \\
\hline 70 & PHLDA3 \\
\hline 71 & $B M P 7$ \\
\hline 72 & SERPINF1 \\
\hline 73 & $A D D 3$ \\
\hline 74 & VPS13D \\
\hline 75 & MST1 \\
\hline 76 & USP3 \\
\hline 77 & SERINC5 \\
\hline 78 & LRP5 \\
\hline 79 & $F A M 46 A$ \\
\hline 80 & $P A 2 G 4$ \\
\hline 81 & AIMI \\
\hline 82 & TIA1 \\
\hline 83 & SYT11 \\
\hline 84 & МАРК 10 \\
\hline 85 & IGFBP6 \\
\hline 86 & $K L F 4$ \\
\hline 87 & FAM131B \\
\hline 88 & $C 1 R$ \\
\hline 89 & FSD1 \\
\hline 90 & $C A D M 1$ \\
\hline 91 & SYT11 \\
\hline 92 & $A D I R F$ \\
\hline 93 & R3HCC1 \\
\hline 94 & SMC6 \\
\hline 95 & PIK3R1 \\
\hline 96 & $A R R B 1$ \\
\hline 97 & SLC48A1 \\
\hline 98 & TNFRSF $10 B$ \\
\hline 99 & $D Y R K 2$ \\
\hline 100 & MYO7A \\
\hline
\end{tabular}


Table II. Clusters identified by the mAP-KL method for fibroids.

\begin{tabular}{|c|c|c|}
\hline Cluster & No. of genes & Genes \\
\hline 1 & 31 & $\begin{array}{l}\text { NAV2, CALCOCO2, NPDC1, PLAT, RPL38, COPS8, LMBRD1, PCNA, SERPINF1, SERINC5, } \\
\text { KLF4, C1R, ADIRF, R3HCC1, FNBP1L, RTN3, YARS, ABLIM1, IVNS1ABP, TRPS1, ADD3, } \\
\text { C1S, IFT20, UBE2L6, SMARCA2, LOXL2, SUCLG2, RPL38, BCAM, H1FX, MAFB }\end{array}$ \\
\hline 2 & 12 & COL4A1, COL4A2, PDGFC, COL5A2, FN1, COL3A1, PALLD, SOX4, CST3, CD74, SPARC, FN1 \\
\hline 3 & 21 & $\begin{array}{l}\text { HEPH, ANXA1, PIK3R1, COPS8, ITM2C, C9orf3, DCHS1, ALDH2, CYR61, LGALS3, PXDN, } \\
\text { FBXL7, ADGRE5, PIK3R1, FYN, SMS, ENAH, SPCS3, SLC24A3, GNG11, CYR61 }\end{array}$ \\
\hline 4 & 18 & $\begin{array}{l}\text { SNCG, HTATIP2, DPT, SULT1A2, KLF4, AIM1, ARRB1, SLC48A1, NPR1, TPSAB1, HFE, RAC2, } \\
\text { RPS11, TOE1, SLC7A6, TESC, VRK3, SERPINB1 }\end{array}$ \\
\hline 5 & 33 & $\begin{array}{l}\text { KIF5C, CAPN6, EPB41L2, MAP4K4, ADGRL1, VAMP2, SAV1, TEX10, IRF2, PRKD1, GCN1, } \\
\text { PHLDA3, ADD3, USP3, FAM46A, PA2G4, TIA1, SYT11, MAPK10, IGFBP6, SMC6, } \\
\text { TNFRSF10B, DYRK2, LRP12, NUDT3, TFAP2C, INPP1, ABCC1, EC12, FLOT2, PPARD, GPSM2, } \\
\text { TFPI }\end{array}$ \\
\hline 6 & 13 & $\begin{array}{l}\text { BAX, GPSM2, TIMM44, UST, COL9A2, LRP5, CADM1, FAAP100, AEN, TTC27, GSTM5, } \\
\text { KNOP1, C1QL1 }\end{array}$ \\
\hline 7 & 20 & $\begin{array}{l}\text { IGSF3, KCNS3, POPDC2, GATA2, PCBP2, SULT1A1, FAM131B, SYT11, NRN1, ENOX2, } \\
\text { NUDT11, TDRD7, MTCL1, ALDH1A1, RARRES3, MARK3, ST3GAL2, ST3GAL1, SLC46A3 }\end{array}$ \\
\hline 8 & 22 & $\begin{array}{l}\text { FMO4, ABLIM3, CAMKMT, KCNN1, FAM63B, BCAN, CBX8, PRR5L, PARVB, ACAN, BMP7, } \\
\text { VPS13D, FSD1, MYO7A, CDK18, TMEM59L, FLT3LG, NPR1, PLCE1, BTNL3, POLG, CSPP1 }\end{array}$ \\
\hline 9 & 18 & $\begin{array}{l}\text { SYBU, BCAN, RTP4, PHYHIP, MST1, PEX7, TMEM246, EGFR, MST1, PTK2B, TBC1D13, } \\
\text { RALGDS, SP140L, ZMYND8, CCDC22, CLCN7, PIDD1, NKX3-1, }\end{array}$ \\
\hline
\end{tabular}

data point as a node in a network and recursively transmits real-valued messages along edges of the network until a good set of exemplars and corresponding clusters emerge (2). In this final step, $n$ ( $n=k$, the KL index) clusters were explored among the top $N$ genes according to the pre-defined number, and a list of $n$ exemplars was obtained (18). These were expected to form a classifier to discriminate between the normal and fibroid classes in a test set that was formulated through retaining only those $n$ genes required to proceed with the classification.

Classification and evaluation. In the present study, SVM (19) with linear kernel (20) was applied to evaluate the performance of mAP-KL. Initially, a five-fold cross-validation was conducted on the training set to evaluate the potential classification strength of the models, and then its prediction was computed on a separate test set.

For the purpose of accessing the classification results, several measures were selected, which included the area under the receiver operating characteristics curve (AUC), the true negative rate (TNR), true positive rate (TPR) and the Matthews coefficient correlation classification (MCC). In detail, accuracy (ACC) was one of the most popular performance measures in machine learning classification; however, it did not take into account the nature of the incorrect predictions. Therefore, the AUC was used, as it had been introduced as a better measure for evaluating the predictive ability of machine learners compared with ACC (21). Additionally, TNR or specificity represented the ratio of correctly classified negatives to the actual number of negatives, as well as TPR or sensitivity, which was defined to be the ratio of positives correctly classified to the actual number of positives (22). Furthermore, MCC was a measure of the quality of binary classification and considered the true and false positive and negatives (23). The combination of those measures provided an adequate overview of the classification's performance.

MIN construction and topological analysis

MIN construction. MIN typically relied on the estimation of mutual information (MI) between all pairs of variables, and has previously attracted the attention of the bioinformatics community for the inference of very large networks $(24,25)$. MIN construction for cluster related top $N$ genes was comprised of three steps. The first step was the computation of the MI matrix (MIM), a square matrix whose $i, j$-th element was the mutual information between the random genes $X_{i}$ and $X_{j}$, and $q$ was a probability measure.

$$
M I M_{i j}=I\left(X_{i} ; X_{j}\right)=\sum_{i, j} q\left(x_{i}, y_{j}\right) \log \frac{q\left(x_{i}, y_{j}\right)}{q\left(x_{i}\right) q\left(y_{j}\right)}
$$

The second step was the computation of an edge score for each pair of nodes by the algorithm. The CLR algorithm was an extension of the relevance network approach (25) and the MI for each pair of genes was computed and a score related to the empirical distribution of the MI values was derived (26). In particular, instead of considering the information $I\left(X_{i} ; X_{j}\right)$ between genes $X_{i}$ and $X_{j}$, it took into account the edge score:

$$
\begin{aligned}
& z_{i j}=\sqrt{z_{i}^{2}+z_{j}^{2}} \\
& \text { Where } z_{i}=\max \left(0, \frac{I\left(X_{i} ; X_{j}-\mu_{i}\right)}{\sigma_{i}}\right)
\end{aligned}
$$




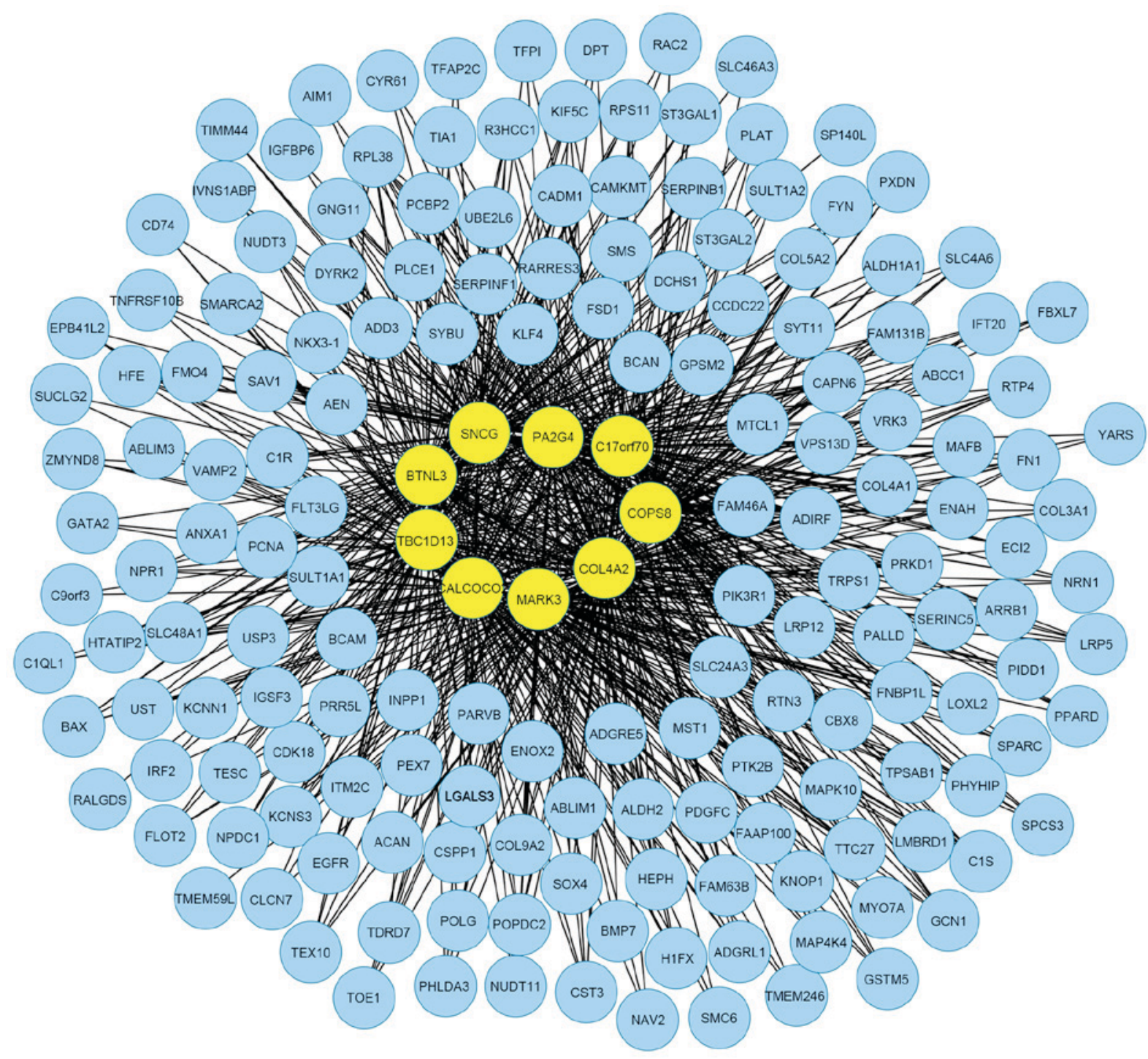

Figure 2. Mutual information network for clusters related the top 200 genes of fibroid. There were 174 nodes and 820 edges, where nodes represented genes, and edges were the interactions between two genes. The yellow nodes, CALCOCO2, COL4A2, COPS8, SNCG, PA2G4, C17orf70, MARK3, BTNL3 and TBC1D13, stood for exemplars of nine clusters identified via the multiple affinity propogation-Krzanowski and Lai method.

Of which $\mu_{i}$ and $\sigma_{i}$ represented the sample mean and standard deviation of the empirical distribution of the values $I\left(X_{i}\right.$; $\left.X_{j}\right)$, respectively.

The final step was to input the genes and edge scores into the igraph software package (27) and to visualize the MIN.

Topological analysis of MIN. In order to investigate the biological functions and significance of nodes in the MIN, the importance was characterized using indices of topological analysis [degree (28), closeness (29), betweenness (30) and transitivity (31)]. The degree quantifies the local topology of each gene by summing up the number of its adjacent genes. Closeness centrality was a measure of the average length of the shortest paths to access all other genes in the network. Betweenness centrality was a shortest paths enumeration-based metric in graphs for determining how the neighbors of a node were interconnected, and was considered the ratio of the node in the shortest path between two other nodes. Furthermore, transitivity, a measure for clustering coefficient, gave an indication of the clustering in the whole network.

\section{Results}

In the present study, the top 200 genes in the gene expression profile were reserved based on maxT multiple hypothesis testing for further exploitation, and the top 100 genes are listed in Table I. Following the AP-KL, an elaborate set of analytical clusters with SVM on the training set was executed, and validation was conducted on a separate test set in order to evaluate its performance across the gene expression data. Meanwhile, MIN for the top 200 genes was constructed and its topological properties were identified in order to investigate the roles and significance of cluster genes and exemplars obtained from mAP-KL in fibroid.

Clusters and exemplars. By performing the AP clustering algorithm in conjunction with KL cluster quality for the top 200 genes, the quantity of clusters with $<50$ genes was 9 , whose gene compositions are displayed in Table II. In addition, the gene compositions of different clusters were varied, and amongst them cluster 5 contained the highest number of genes 
Table III. Topological properties of exemplars in mutual information network.

\begin{tabular}{lrcrr}
\hline Exemplar & Degree & Closeness & Betweenness & Transitivity \\
\hline CALCOCO2 & 104 & 174.81 & 3.54 & 1022 \\
COL4A2 & 110 & 237.21 & 3.71 & 1075 \\
COPS8 & 88 & 147.27 & 3.29 & 657 \\
SNCG & 127 & 263.29 & 3.89 & 1122 \\
PA2G4 & 91 & 152.57 & 1.83 & 35 \\
C17orf70 & 155.03 & 0.50 & 0 \\
MARK3 & 85 & 149.75 & 3.05 & 44 \\
BTNL3 & 93 & 131.67 & 3.01 & 0 \\
TBC1D13 & 87 & 158.98 & 3.55 & 897
\end{tabular}

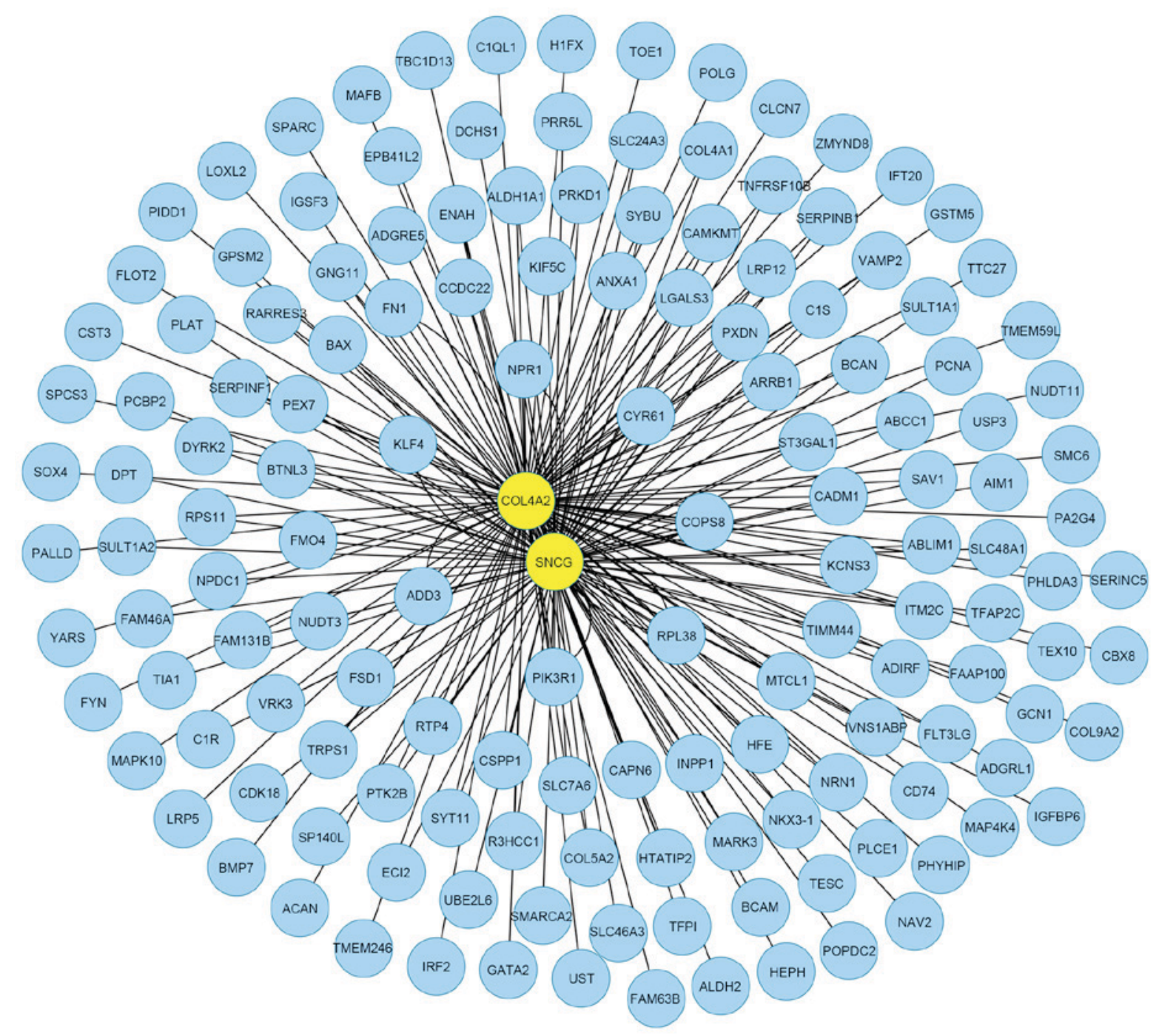

Figure 3. Sub-network for key genes (SNCG and COL4A2) extracted from the mutual information network, in which a total of 149 nodes and 222 edges were mapped. Nodes represented genes, and edges were the interactions between two genes. The yellow nodes were key genes of fibroid.

(33 genes), whereas, only 12 genes were present in cluster 2 . Furthermore, for each cluster, an exemplar was detected on the basis of the AP clustering method, which may be important in the progress of fibroid. From cluster 1 to 9 , their exemplars were CALCOCO2, COL4A2, COPS8, SNCG, PA2G4, C17orf70, MARK3, BTNL3 and TBC1D13, respectively.
Evaluation by the SVM model. The test set consisted of the 9 exemplars. SVM with linear kernel was used to possess the performance of mAP-KL. This method achieved the highest classification scores $(\mathrm{AUC}=1.00, \mathrm{TNR}=1.00, \mathrm{TPR}=1.00$ and $\mathrm{MCC}=1.00)$; therefore, it was concluded that the classification results were almost ideal during the SVM evaluation, 


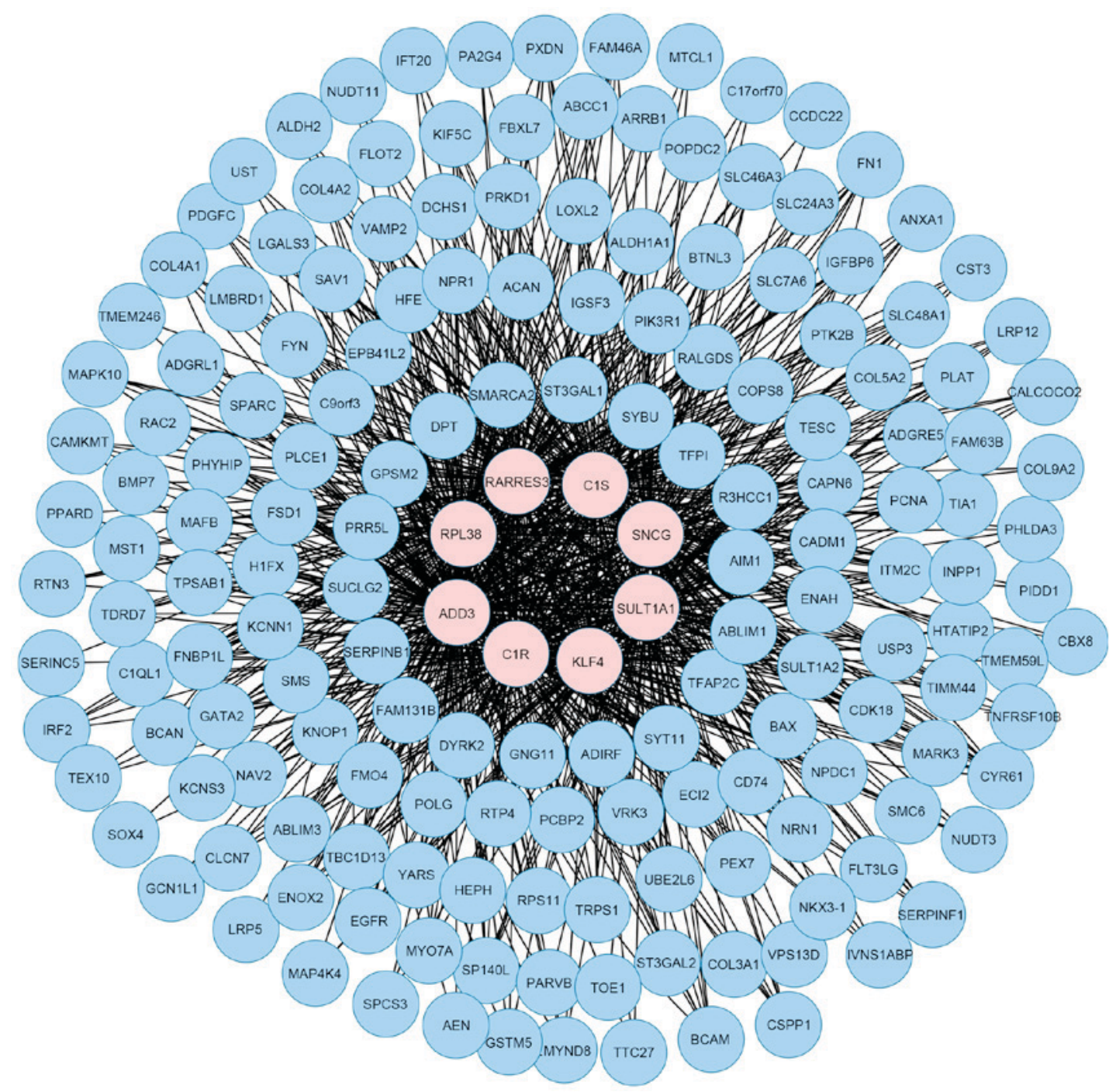

Figure 4. MIN for the top 200 ranked genes in the microarray data. There were 174 nodes and 1,002 interactions, where nodes represented genes, and edges were the interactions between two genes. The pink nodes were hub genes with top 5\% degree distribution of the MIN. MIN, mutual information network.

and inferred that the mAP-KL methodology that combined ranking-filtering and cluster analysis was feasible and suitable for identifying exemplars of fibroid.

Topological analysis of MIN. The ideal evaluation of the classification for the mAP-KL method gave more confidence to the significance of 9 exemplars in fibroid, whereas those were also expected to form a classifier or module that is distinct between the normal controls and fibroid patients. Therefore, an MIN was constructed for the top 200 genes associated with cluster genes. A total of 178 genes were mapped to the MIN and 820 interactions were observed (Fig. 2). The topological properties of the 9 exemplars in the MIN are shown in Table III. The results for exemplars were inconsistent due to different methods, except for $S N C G$ and $C O L 4 A 2$. Notably, the degree, closeness, betweenness and transitivity of $S N C G$ were the highest among exemplars with 127, 263.29, 3.89 and 1122 , separately. The second highest results were observed in COL4A2 with a degree, closeness, betweenness and transitivity of 110, 237.21, 3.71 and 1,075, respectively. Therefore, the two exemplars were more important for fibroid, thus we mined their connections from the MIN. The two genes were able to interact with each other and build a small module (Fig. 3) with 149 genes and 222 interactions. Furthermore, a MIN with 174 nodes and 1,002 interactions for the top 200 ranked genes in the microarray data was demonstrated in Fig. 4.

\section{Discussion}

Fibroid, also known as uterine leiomyoma or uterine fibroid, is begin smooth muscle neoplasm of the uterus and the most widespread gynecological problem amongst women (32). Important symptoms of fibroid include abnormal uterine bleeding, heavy or painful menstrual periods, abdominal discomfort or bloating, painful defecation, back ache, urinary frequency or retention, and in some cases, infertility (33). Although surgical staging and nomograms may help predict the clinical outcome, the five-year survival rate for uterus-confined disease remains $<50 \%$ (34). Therefore, understanding the molecular biology and investigating potential biomarkers of fibroid may provide further prognostic and therapeutic insights.

In the present study, nine exemplars were investigated for clusters, which were obtained from the top 200 genes via the mAP-KL method, including CALCOCO2, COL4A2, COPS8, 
SNCG and PA2G4. Subsequently, the SVM model was used to assess the performance of the mAP-KL method to classify normal controls and fibroid patients, and the results demonstrated that there was a good classification, which indicated the feasibility of the mAP-KL method to identify exemplars in fibroid. In order to further explore the biological significance of exemplars, the topological analysis (degree, closeness, betweenness and transitivity) for MIN constructed by the CLR algorithm were conducted and two key genes, $S N C G$ and COL4A2, were obtained resulting from their better topological properties compared with the other seven genes.

Constructing the MIN for the cluster related top 200 genes and building a network for them allowed for the determination of the activities of the genes in the network. Fig. 4 shows the MIN for the 200 top ranked genes in the microarray data, but four types of topological properties had no intersections and it may be inferred that this network was not as strict as the cluster MIN. In general, the degree was a familiar index, thus genes with a quantile of the top $5 \%$ degree distributions were defined as hub genes. Therefore, 8 hub genes were obtained in total, which included RARRES3, RPL38, ADD3, CIR, $K L F 4, S U L T 1 A 1, S N C G$ and $C 1 S$. In addition, there were great differences between exemplars and hub genes, except for one common gene, $S N C G$.

$S N C G$ is a member of the synuclein neuronal protein family along with $S N C A$ and $S N C B$, and is highly tissue-specific. In addition, it is expressed at presynaptic terminals in the brain and the peripheral nervous system (35). It promotes migration, invasion and metastasis of tumor cells (36), which have proven that expression of $S N C G$ protein is elevated in the advanced stages of various types of cancers, including ovarian (37), lung, liver (38), esophagus (39), colon (38) and in particular, breast $(40,41)$. For example, Singh and Jia (41) revealed that $S N C G$ protein expression was correlated with pathological factors, including lymph node metastasis, tumor size and stage, and follow-up revealed that the survival rate of patients with $S N C G$-positive protein expression were significantly lower than the patients with negative expression for 438 cases with breast cancer. Furthermore, it had been demonstrated that overexpression of $S N C G$ was a predictor for aggressive tumor behavior and adverse outcome in patients with endometrial cancer (42), and was a prognostic tool and therapeutic target in uterine serous carcinoma (43). However, there is currently no published research reporting the association between $S N C G$ and fibroids, although it correlated with other uterine-associated cancers as described above. To the best of our knowledge the present study proposed for the first time that $S N C G$ acts as a key role in fibroid.

COL $4 A 2$ was the other key gene for fibroids, which was implicated in extracellular matrix formation and encoded for collagen $\alpha$, which is one of the main components of the endothelial basement membrane (44). Previous research reported that $C O L 4 A 2$ was overexpressed in fibroid, and that it displayed an anti-angiogenic gene expression profile in fibroids when compared with adjacent normal myometrium. These observations may explain the reduced microvascular density observed in fibroids relative to the myometrium (45) and was in accordance with the reduced microvessel density based on anti-von Willebrand factor immunostaining that was observed in fibroid with respect to normal myometrium (46). Additionally, Gilden et al (47) suggested that protein expression of $C O L 4 A 2$, versican and fibromodulin was increased in untreated leiomyoma cells compared with untreated patient-matched myometrial cells. Therefore, COL4A2 was differentially expressed between normal controls and fibroids.

In conclusion, nine exemplars were identified based on the mAP-KL method, and two key genes (SNCG and COL4A2) were investigated according to a topological analysis of MIN. These genes may be potential biomarkers for the detection and therapy of fibroids. Furthermore, the present study may give an insight for future studies associated with fibroid.

\section{References}

1. Frey BJ and Dueck D: Clustering by passing messages between data points. Science 315: 972-976, 2007.

2. Bodenhofer U, Kothmeier A and Hochreiter S: APCluster: An R package for affinity propagation clustering. Bioinformatics 27: 2463-2464, 2011.

3. Kiddle SJ, Windram OP, McHattie S, Mead A, Beynon J, Buchanan-Wollaston V, Denby KJ and Mukherjee S: Temporal clustering by affinity propagation reveals transcriptional modules in Arabidopsis thaliana. Bioinformatics 26: 355-362, 2010.

4. Woźniak M, Tiuryn J and Dutkowski J: MODEVO: Exploring modularity and evolution of protein interaction networks. Bioinformatics 26: 1790-1791, 2010.

5. Wang CW, Chen KT and Lu CL: iPARTS: An improved tool of pairwise alignment of RNA tertiary structures. Nucleic Acids Res 38 (Web Server issue): W340-W347, 2010.

6. Sakellariou A, Sanoudou D and Spyrou G: Combining multiple hypothesis testing and affinity propagation clustering leads to accurate, robust and sample size independent classification on gene expression data. BMC bioinformatics 13: 270, 2012.

7. Krzanowski WJ and Lai Y: A criterion for determining the number of groups in a data set using sum-of-squares clustering. Biometrics 44: 23-34, 1988.

8. Liu ZP, Wang Y, Zhang XS and Chen L: Network-based analysis of complex diseases. IET Syst Biol 6: 22-33, 2012.

9. Chen L, Wang RS and Zhang XS: Reconstruction of Gene Regulatory Networks. In: Biomolecular Networks John Wiley \& Sons, Inc., pp47-87, 2009.

10. Wylie D, Shelton J, Choudhary A and Adai AT: A novel mean-centering method for normalizing microRNA expression from high-throughput RT-qPCR data. BMC Res Notes 4: 555, 2011.

11. Cheadle C, Vawter MP, Freed WJ and Becker KG: Analysis of microarray data using Z score transformation. J Mol Diagn 5: 73-81, 2003.

12. Bolstad BM, Irizarry RA, Astrand M and Speed TP: A comparison of normalization methods for high density oligonucleotide array data based on variance and bias. Bioinformatics 19: 185-193, 2003.

13. Ballman KV, Grill DE, Oberg AL and Therneau TM: Faster cyclic loess: Normalizing RNA arrays via linear models. Bioinformatics 20: 2778-2786, 2004.

14. Pollard K, Dudoit S and van der Laan M: Multiple testing procedures: R multtest package and applications to genomics. UC Berkeley Division of Biostatistics Working Paper Series. Working Paper 164. http://www.bepress.com/ucbbiostat/paper164, 2004.

15. Westfall PH and Young SS (eds): Resampling-Based Multiple Testing: Examples and Methods for P-Value Adjustment. John Wiley \& Sons, New York, 1993.

16. Sakellariou A, Sanoudou D and Spyrou G: Investigating the minimum required number of genes for the classification of neuromuscular disease microarray data. IEEE Trans Inf Technol Biomed 15: 349-355, 2011.

17. Jeffery IB, Higgins DG and Culhane AC: Comparison and evaluation of methods for generating differentially expressed gene lists from microarray data. BMC Bioinformatics 7: 359, 2006.

18. R Development Core Team: R: A Language and Environment for Statistical Computing. R Foundation for Statistical Computing, Vienna, 2012.

19. Chang CC and Lin CJ: LIBSVM: A library for support vector machines. ACM Transactions on Intelligent Systems and Technology (TIST) 2: 27, 2011. 
20. Aha DW, Kibler D and Albert MK: Instance-based learning algorithms. Machine Learning 6: 37-66, 1991.

21. Huang J and Ling CX: Using AUC and accuracy in evaluating learning algorithms. IEEE Transactions on Knowledge and Data Engineering 17: 299-310, 2005.

22. Baldi P, Brunak S, Chauvin Y, Andersen CA and Nielsen $\mathrm{H}$ Assessing the accuracy of prediction algorithms for classification: An overview. Bioinformatics 16: 412-424, 2000.

23. Kantardjieff KA and Rupp B: Matthews coefficient probabilities: Improved estimates for unit cell contents of proteins, DNA and protein-nucleic acid complex crystals. Protein Sci 12: 1865-1871, 2003.

24. Butte AJ and Kohane IS: Mutual information relevance networks: Functional genomic clustering using pairwise entropy measurements. Pac Symp Biocomput 418-429, 2000.

25. Faith JJ, Hayete B, Thaden JT, Mogno I, Wierzbowski J, Cottarel G, Kasif S, Collins JJ and Gardner TS: Large-scale mapping and validation of Escherichia coli transcriptional regulation from a compendium of expression profiles. PLoS Biol 5: e8, 2007.

26. Meyer PE, Lafitte F and Bontempi G: Minet: AR/Bioconductor package for inferring large transcriptional networks using mutual information. BMC Bioinformatics 9: 461, 2008.

27. Csardi G and Nepusz T: Igraph-package. 2008. URL http://cneurocvs.rmki.kfki.hu/igraph/doc/R/aaa-igraph-package. html.

28. Haythornthwaite C: Social network analysis: An approach and technique for the study of information exchange. Library \& Information Science Research 18: 323-342, 1996.

29. Wasserman S: Social network analysis: Methods and applications. Cambridge Univ Press 25: 11, 1994.

30. Barthelemy $\mathrm{M}$ : Betweenness centrality in large complex networks. Eur Physical J B 38: 163-168, 2004.

31. Schank T and Wagner D: Approximating clustering-coefficient and transitivity. J Graph Algorithms Applications 9: 265-275, 2005.

32. Islam MS, Protic O, Giannubilo SR, Toti P, Tranquilli AL, Petraglia F, Castellucci M and Ciarmela P: Uterine leiomyoma: Available medical treatments and new possible therapeutic options. J Clin Endocrinol Metab 98: 921-934, 2013.

33. Bulun SE: Uterine fibroids. N Engl J Med 369: 1344-1355, 2013

34. Zivanovic O, Jacks LM, Iasonos A, Leitao MM Jr, Soslow RA Veras E, Chi DS, Abu-Rustum NR, Barakat RR, Brennan MF and Hensley ML: A nomogram to predict postresection 5-year overall survival for patients with uterine leiomyosarcoma. Cancer 118: 660-669, 2012

35. Lavedan C, Leroy E, Dehejia A, Buchholtz S, Dutra A, Nussbaum RL and Polymeropoulos MH: Identification, localization and characterization of the human gamma-synuclein gene. Hum Genet 103: 106-112, 1998.
36. Zhuang Q, Liu C, Qu L and Shou C: Synuclein- $\gamma$ promotes migration of MCF7 breast cancer cells by activating extracellular-signal regulated kinase pathway and breaking cell-cell junctions. Mol Med Rep 12: 3795-3800, 2015.

37. Mori K, Akers S, Bshara W, et al: Gamma synuclein expression in ovarian cancer. Gynecol Oncol 131: 272, 2013.

38. Guo C, Sun L, Liu Q, Xie YB and Wang X: SNCG expression and clinical significance in colorectal cancer liver metastasis. Zhonghua Wei Chang Wai Ke Za Zhi 15: 625-628, 2012 (In Chinese).

39. Shi L, Zheng S, Zhao Y, Wang JW and Zhan XH: Expressions of SNCG in gastric adenocarcinoma and its clinical significances. Pro Mod Bio 11: 1532-1535, 2011.

40. Min L, Ma R, Yuan H, Liu CY, Dong B, Zhang C, Zeng Y, Wang L, Guo JP, Qu LK, et al: Combined expression of metastasis related markers Naa10p, SNCG and PRL-3 and its prognostic value in breast cancer patients. Asian Pac J Cancer Prev 16: 2819-2826, 2015.

41. Singh VK and Jia Z: Targeting synuclein-gamma to counteract drug resistance in cancer. Expert Opin Ther Targets 12: 59-68, 2008.

42. Mhawech-Fauceglia P, Wang D, Syriac S, Godoy H, Dupont N, Liu S and Odunsi K: Synuclein- $\gamma$ (SNCG) protein expression is associated with poor outcome in endometrial adenocarcinoma. Gynecol Oncol 124: 148-152, 2012.

43. Morgan J, Hoekstra AV, Chapman-Davis E, Hardt JL, Kim JJ and Buttin BM: Synuclein-gamma (SNCG) may be a novel prognostic biomarker in uterine papillary serous carcinoma. Gynecol Oncol 114: 293-298, 2009

44. Kuo DS, Labelle-Dumais C and Gould DB: COL4A1 and COL4A 2 mutations and disease: Insights into pathogenic mechanisms and potential therapeutic targets. Hum Mol Genet 21: R97-R110, 2012

45. Weston G, Trajstman AC, Gargett CE, Manuelpillai U, Vollenhoven BJ and Rogers PA: Fibroids display an anti-angiogenic gene expression profile when compared with adjacent myometrium. Mol Hum Reprod 9: 541-549, 2003.

46. Poncelet C, Fauvet R, Feldmann G, Walker F, Madelenat P and Darai E: Prognostic value of von Willebrand factor, CD34, CD31, and vascular endothelial growth factor expression in women with uterine leiomyosarcomas. J Surg Oncol 86: 84-90, 2004.

47. Gilden M, Malik M, Britten J, Delgado T, Levy G and Catherino WH: Leiomyoma fibrosis inhibited by liarozole, a retinoic acid metabolic blocking agent. Fertil Steril 98: 1557-1562, 2012 . 